This item was submitted to Loughborough's Research Repository by the author.

Items in Figshare are protected by copyright, with all rights reserved, unless otherwise indicated.

\title{
New normalized constant modulus algorithms with relaxation
}

PLEASE CITE THE PUBLISHED VERSION

PUBLISHER

(C) IEEE

VERSION

VoR (Version of Record)

LICENCE

CC BY-NC-ND 4.0

\section{REPOSITORY RECORD}

Tanrikulu, Oguz, A.G. Constantinides, and Jonathon Chambers. 2019. "New Normalized Constant Modulus Algorithms with Relaxation”. figshare. https://hdl.handle.net/2134/5864. 
This item was submitted to Loughborough's Institutional Repository (https://dspace.lboro.ac.uk/) by the author and is made available under the following Creative Commons Licence conditions.

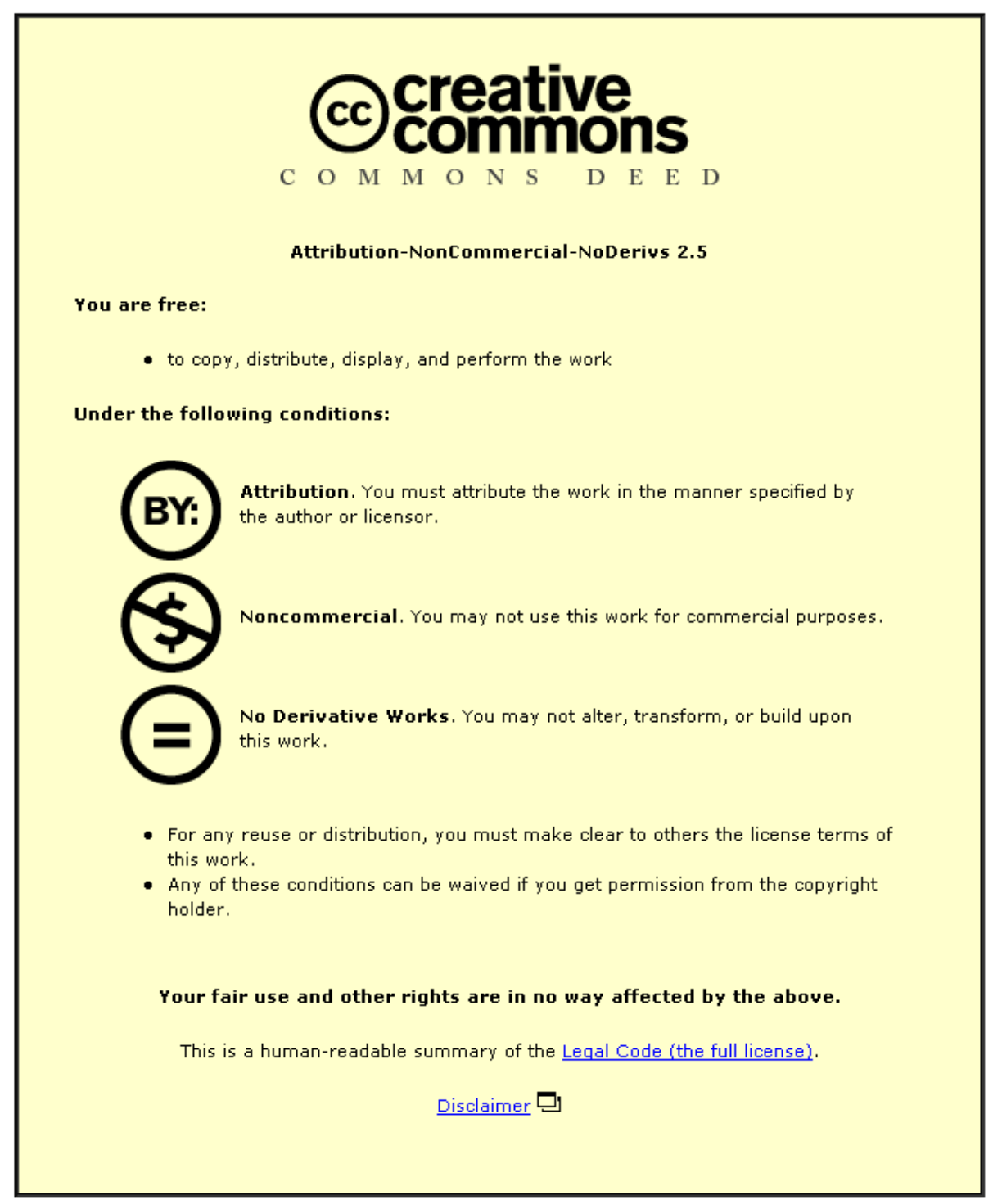

For the full text of this licence, please go to: http://creativecommons.org/licenses/by-nc-nd/2.5/ 


\title{
New Normalized Constant Modulus Algorithms with Relaxation
}

\author{
Oğuz Tanrıkulu, Member, IEEE, Anthony G. Constantinides, Senior Member, IEEE, \\ and Jonathon A. Chambers, Member, IEEE
}

\begin{abstract}
A new normalized constant modulus algorithm is proposed that has a more desirable error performance surface (EPS) than the existing constant modulus blind equalization algorithms. We show that for an autoregressive channel, a wellknown class of undesirable local solutions (ULS's) does not exist. EPS's and convergence of the parameters are shown for a number of channels for which well-known algorithms are known to possess ULS's.
\end{abstract}

Index Terms - Adaptive equalizers, blind algorithms, stationary points.

\section{INTRODUCTION}

C ONSIDER the baseband representation for digital data transmission in Fig. 1, where $a_{k}$ is the transmitted symbols, $w_{k}$ is the channel noise, $x_{k}$ is the equalizer input, $y_{k}$ is the equalizer output and $\hat{a}_{k}$ is the output of the decision device [1]. The objective of a blind equalization (BE) algorithm is to achieve $\hat{a}_{k}=e^{j \phi} a_{k-\Delta}$ without using a training signal available at the receiver. The equalizer weight vector and the input vector to the equalizer are respectively defined as $\theta_{k}=\left[\theta_{0 \mid k} \theta_{1 \mid k} \cdots \theta_{m \mid k}\right]^{T}$ and $X_{k}^{H}=\left[x_{k} x_{k-1} \cdots x_{k-m}\right]$.

The existing algorithms that are based on minimizing a nonlinear function of the equalizer output, $y_{k}$, possess undesirable local solutions (ULS's) for which the "eye" is left "closed" after convergence. The normalized constant modulus algorithm (NCMA) was introduced in [2] in order to maximize the convergence speed of the Godard algorithm [with $\mu=1$ in (2)], and was developed further in [3]-[5] as a link between blind equalization and classical adaptive filtering where a training (desired) signal, $d_{k}$, is available. In [5], the NCMA is formally derived from the exact solution of the orthodox formulation below

$$
\min _{\theta_{k+1}}\left\{\left|d_{k}-X_{k}^{H} \theta_{k+1}\right|+\left(\frac{1}{\mu}-1\right)\left\|X_{k}\left|\left\|_{2}^{2}|| \theta_{k+1}-\theta_{k}\right\|_{2}^{2}\right\}\right.\right.
$$

for the stepsize $\mu \in(0,1)$ and $d_{k} \in \mathcal{D}$ where $\mathcal{D} \triangleq\{z \in$ $\mathcal{C}:|z|=1\}$, and the corresponding update equation is

$$
\theta_{k+1}=\theta_{k}+\frac{\mu}{\left\|X_{k}\right\|_{2}^{2}}\left[\operatorname{sgn}\left(y_{k}\right)-y_{k}\right] X_{k} \text {. }
$$

Sliding window NCMA algorithms based on constant modulus and decision-directed updates are also provided in [5].

Manuscript received March 3, 1997. The associate editor coordinating the review of this manuscript and approving it for publication was Prof. V. J. Mathews.

The authors are with the Department of Electrical and Electronic Engineering, Imperial College of Science, Technology and Medicine, London SW7 2BT, U.K. (e-mail: o.tanrikulu@ic.ac.uk).

Publisher Item Identifier S 1070-9908(97)06694-7.

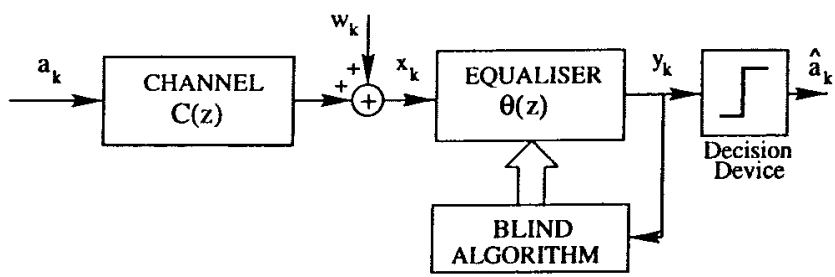

Fig. 1. Blind equalization in the baseband.

The merit of the NCMA is fast convergence speed and the increased likelihood of escaping ULS's for large stepsizes. However, the nature of the error performance surface (EPS) of this algorithm is not vastly different from that of the Sato algorithm which, in many instances, contains a large number of ULS's. An illustrative example is given in the simulations section.

The contribution in this paper is the development of modified NCMA updates [6]-[8] that alleviate the problem of convergence to ULS's. The EPS's of the proposed algorithms are shown to possess much improved characteristics compared to the existing algorithms, including the CMA [1], which is commonly accepted as the most promising adaptive blind equalization algorithm for real-time implementation.

\section{Proposed Algorithm}

We assume a complex, multilevel source signal and formulate the following deterministic optimization problem

$$
\min _{\theta_{k+1}}\{\left\|\theta_{k+1}-\theta_{k}\right\|_{2}^{2}+\lambda \underbrace{\left(X_{k}^{H} \theta_{k+1} \theta_{k+1}^{H} X_{k}-R^{2}\right)}_{\text {constraint }}\}
$$

where $\lambda$ is the Lagrange multiplier and $R$ is a dispersion constant defined in the sequel. We also define

$$
y_{k} \triangleq X_{k}^{H} \theta_{k}, s_{k} \triangleq X_{k}^{H} \theta_{k+1} \text {. }
$$

By differentiating (3) with respect to the real and imaginary parts of $\theta_{k+1}$, one can obtain

$$
\theta_{k+1}-\theta_{k}+\lambda X_{k} X_{k}^{H} \theta_{k+1}=0 .
$$

If (5) is premultiplied by $X_{k}^{H}$, the optimum Lagrange multiplier, $\lambda_{*}$, can be obtained as

$$
\lambda_{*}=-\frac{1}{\left\|X_{k}\right\|_{2}^{2}}\left(1-\frac{y_{k}}{s_{k}}\right)
$$

and the corresponding update equation becomes

$$
\theta_{k+1}=\theta_{k}-\lambda_{*} X_{k} X_{k}^{H} \theta_{k+1} .
$$

The constraint in (3) enforces $s_{k}=X_{k}^{H} \theta_{k+1}=R e^{j \omega_{k}}$ for arbitrary $\omega_{k} \in[-\pi, \pi)$. Furthermore, after premultiplying (7) by 


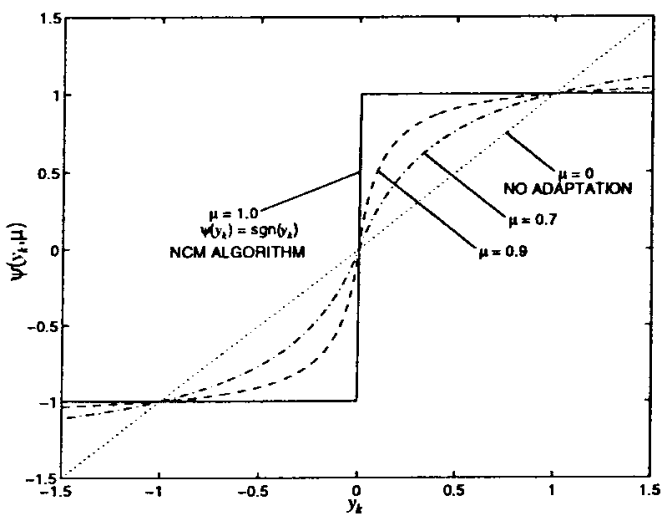

Fig. 2. ZNL of the SCS algorithm.

$X_{k}^{H}$, we see that $s_{k}=R \operatorname{sgn}\left(y_{k}\right)$, where $\operatorname{sgn}\left(r e^{j \omega_{k}}\right) \triangleq e^{j \omega_{k}}$, is the choice corresponding to the exact solution of (3) where the constraint is imposed as a hard constraint. Therefore, the optimal Lagrange multiplier in (6) is

$$
\lambda_{*}=-\frac{1}{\left\|X_{k}\right\|_{2}^{2}}\left(1-\frac{\left|y_{k}\right|}{R}\right) \text {. }
$$

At this point, we deviate from the initial optimization statement in (3), since the continued use of $s_{k}=R \operatorname{sgn}\left(y_{k}\right)$ will inevitably lead to the NCMA update in (2) that possesses ULS's. The nature of the deviation comes after recognizing that once a stepsize is introduced for relaxing the control over the convergence speed, the constraint on $s_{k}$ is retained as a soft constraint unless $\mu=1$. By using (8) in (7), and introducing $\mu$, we have

$$
\theta_{k+1}=\theta_{k}+\frac{\mu}{\left\|X_{k}\right\|_{2}^{2}}\left(1-\frac{\left|y_{k}\right|}{R}\right) X_{k} X_{k}^{H} \theta_{k+1} .
$$

By premultiplying (9) with $X_{k}^{H}$, we obtain

$$
X_{k}^{H} \theta_{k+1}=\frac{y_{k}}{1-\mu\left(1-\frac{\left|y_{k}\right|}{R}\right)} .
$$

Therefore, instead of using $X_{k}^{H} \theta_{k+1}=R \operatorname{sgn}\left(y_{k}\right)$ which is dictated by the criterion in (3), we use (10) in (9) and obtain the update equation of the soft constraint satisfaction (SCS) algorithm as

$$
\begin{aligned}
\theta_{k+1} & =\theta_{k}+\frac{1}{\left\|X_{k}\right\|_{2}^{2}}\left[\psi\left(y_{k}, \mu\right)-y_{k}\right] X_{k} \\
\psi\left(y_{k}, \mu\right) & =\frac{y_{k}}{1-\mu\left(1-\frac{\left|y_{k}\right|}{R}\right)} .
\end{aligned}
$$

Note that, for $\mu=1$ in (11), the SCS algorithm reduces to the NCMA algorithm. Unlike the NCMA, for each $\mu \in(0,1)$, the zero-memory nonlinearity (ZNL) in the SCS algorithm, $\psi\left(y_{k}, \mu\right)$, is different and no hard-limiting is performed at the equalizer output as illustrated in Fig. 2.

The dispersion constant $R$ can be obtained by considering the unnormalized algorithm and an independent identically distributed (i.i.d.) $\left\{a_{k}\right\}$. Around a desirable local solution (DLS), $1-\mu\left(1-\left|y_{k}\right| / R\right) \approx 1$ and following the derivation in [1], it is easy to show that

$$
R=\frac{E\left\{\left|a_{k}\right|^{3}\right\}}{E\left\{\left|a_{k}\right|^{2}\right\}} \text {. }
$$

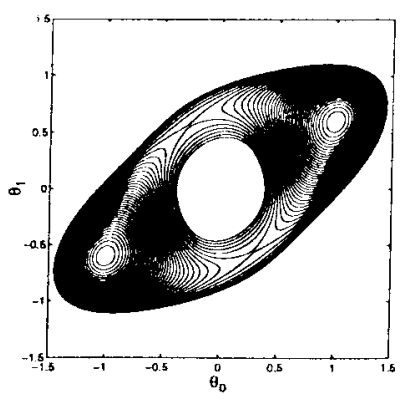

(a)

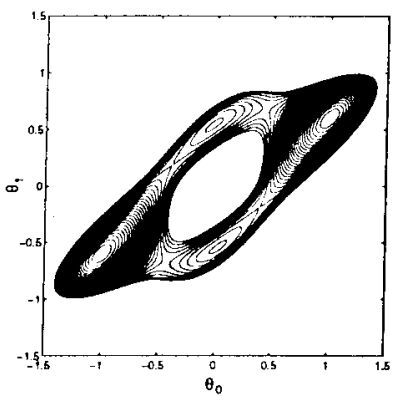

(b)
Fig. 3. EPS's (a) with and (b) without the normalization in (14) for $C_{1}(z)$.

\section{STATIONARY Points}

If $\mu$ is sufficiently small so that $1-\mu|1-| y_{k}|/ R| \approx 1$, then a first-order approximation of the error function in (12) yields the adaptive algorithm

$$
\theta_{k+1}=\theta_{k}+\frac{\mu}{\left\|X_{k}\right\|_{2}^{2}}\left(1-\frac{\left|y_{k}\right|}{R}\right) y_{k} X_{k}
$$

which we denote as the SCS-1 algorithm [9]. The main difference between the SCS and the SCS-1 algorithms has been found to be the speed of convergence rather than the nature and the number of solutions on the EPS. Therefore, for the sake of a tractable analysis, we focus on the SCS-1 algorithm, which is also easier to implement in real time.

Let us use an $\operatorname{AR}(p)$ channel of the form [10]

$$
C(z)=\frac{1}{1+\alpha z^{-p}}, \quad 0<|\alpha|<1
$$

and an FIR equalizer

$$
\theta(z)=\sum_{i=0}^{m} \theta_{i} z^{-i}, \quad m \geq p .
$$

We start by hypothesizing that ULS of the type $\theta_{*}=$ $[00 \cdots \beta]^{T}(\beta \neq 0)$ exists, which commonly occurs in most $\mathrm{BE}$ algorithms [10]. As a stationary point $\theta_{*}$ must satisfy

$$
E\left\{\frac{1}{\left\|X_{k}\right\|_{2}^{2}}\left(1-\frac{\left|y_{k}^{\theta_{*}}\right|}{R}\right) y_{k}^{\theta_{*}} X_{k}\right\}=\mathbf{0}
$$

where $y_{k}^{\theta_{*}}=x_{k-m} \beta$. In other words

$$
E\left\{\frac{1}{\left\|X_{k}\right\|_{2}^{2}}\left(1-\frac{\left|x_{k-m}\right||\beta|}{R}\right) x_{k-m} x_{k-i}^{*}\right\} \beta=0
$$

for $i \in\{0, \cdots, m\}$. Due to (15), $x_{k}, \cdots, x_{k-p+1}$ are statistically independent for i.i.d. channel input [10]. If the normalization $\left\|X_{k}\right\|_{2}^{2}$ is not used, using this independence, the system of equations in (18) would reduce to a single equation (for statistically stationary $x_{k}$ ), which yields

$$
|\beta|=R \frac{E\left\{\left|x_{k}\right|^{2}\right\}}{E\left\{\left|x_{k}\right|^{3}\right\}} .
$$

However, in SCS-1, due to the correlation between the terms in the normalization and $x_{k-m} x_{k-i}^{*}$, we have the following $m+1$ equations:

$$
\begin{gathered}
|\beta|_{i}=R \frac{E\left\{\left(\| X_{k}||_{2}^{2}\right)^{-1} x_{k-m} x_{k-i}^{*}\right\}}{E\left\{\left(\| X_{k}||_{2}^{2}\right)^{-1}\left|x_{k-m}\right| x_{k-m} x_{k-i}^{*}\right\}}, \\
i \in\{0, \cdots, m\} .
\end{gathered}
$$




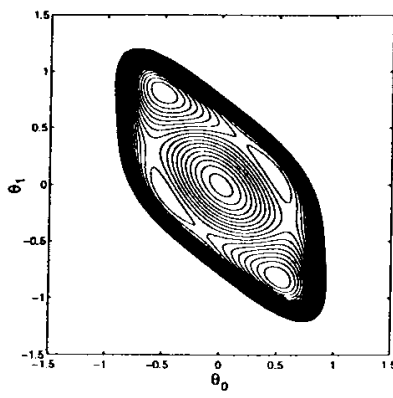

(a)

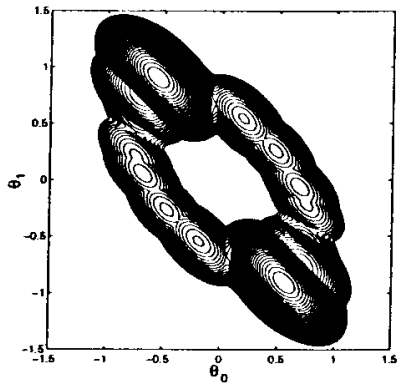

(c)

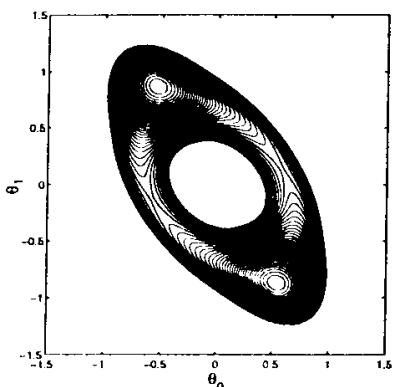

(b)

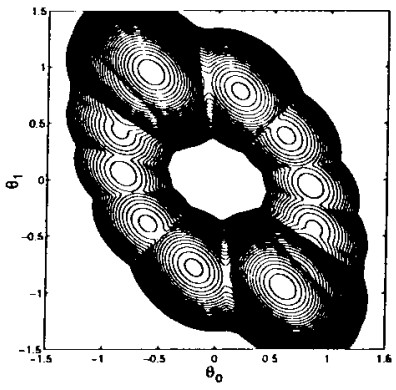

(d)

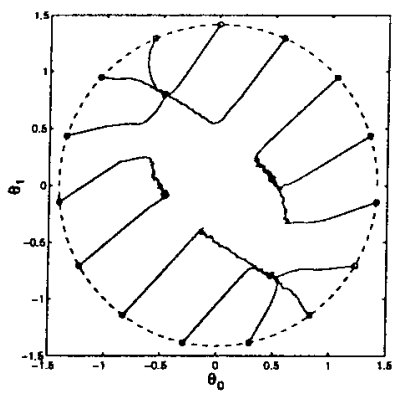

(a)

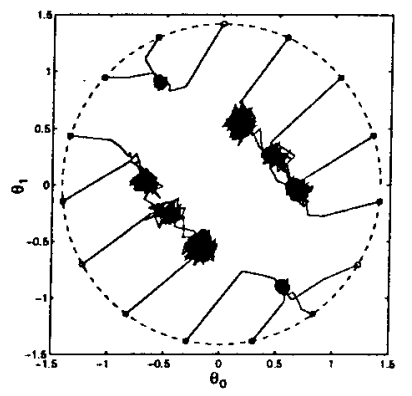

(c)

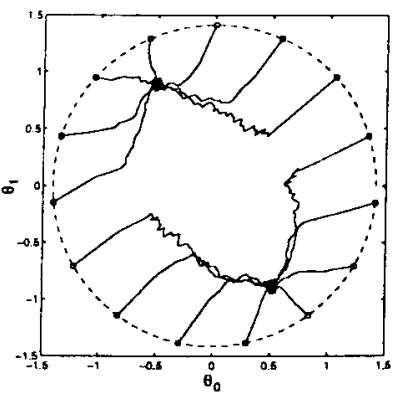

(b)

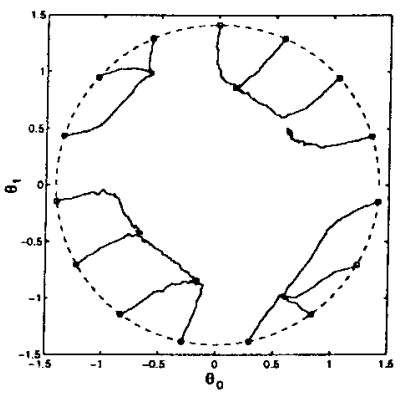

(d)
Fig. 4. EPS's of (a) Godard, (b) SCS-1, (c) Sato, and (d) NCMA for $C_{2}(z)$.

In general, $|\beta|_{i}$ are not equal and, hence, the SCS-1 does not have ULS of the type $\theta_{*}=[00 \cdots \beta]^{T}[8]$ as demonstrated in the next section.

\section{Simulations}

The following channels are used to demonstrate the properties of the SCS-1 algorithm with $\theta=\left[\theta_{0} \theta_{1}\right]^{T}$ :

$$
\begin{aligned}
C_{1}(z) & =\frac{1}{1+0.60 z^{-1}}, \\
C_{2}(z) & =0.49+0.70 z^{-1}+z^{-2} .
\end{aligned}
$$

In Fig. 3, for $C_{1}(z)$, there are no ULS's for the SCS-1. However, if normalization of the gradient vector is omitted in (14), two ULS's appear around $\theta_{*} \approx \pm[00.6]$ as given by (19). The EPS's corresponding to $C_{2}(z)$, which is a maximumphase channel, are shown in Fig. 4. All algorithms except the SCS-1 have ULS's. Finally, the signal-to-noise ratio (SNR) at the channel output is chosen to be $20 \mathrm{~dB}$, and the results are shown Fig. 5. Depending upon the initialization, the NCMA, Godard and Sato algorithms converge to ULS's, but the SCS-1 algorithm converges to DLS's consistently.

\section{CONCLUSIONS}

New normalized constant modulus algorithms are presented that demonstrate more favorable EPS characteristics than the well-known algorithms in the same class. The absence of a class of ULS's is shown for an all-pole channel. Research is underway to generalize the properties of the proposed algorithm for moving average channels and to extend this algorithm to the fractionally spaced and decision-feedback
Fig. 5. Convergence trajectories of (a) Godard, (b) SCS-1, (c) Sato, and (d) NCMA for $C_{2}(z)$.

configurations. Other areas that are currently being explored are the stability region for $\mu$ and improving the convergence speed and the tracking performance by imposing multiple constraints and further modifications to the algorithm.

\section{REFERENCES}

[1] N. Godard, "Self-recovering equalization and carrier tracking in twodimensional data communications systems," IEEE Trans. Commun., vol. COM-28, pp. 1867-1875, Nov. 1980.

[2] K. Hilal and P. Duhamel, "A convergence study of the constant modulus algorithm leading to a normalized-CMA and block-normalized-CMA," in Proc. EUSIPCO'92, Brussels, Belgium, Aug. 1992, pp. 135-138.

[3] C. B. Papadias and D. T. M. Slock, "New adaptive blind equalization algorithms for constant modulus constellations," in Proc. ICASSP'94, vol. 3, pp. 321-324.

[4] C. Papadias, "Methodés d'égalization et d'identification aveugle pour les canaux linéaires," Ph.D. dissertation, Ecole Nationale Supérieure

[5] C. B. Papadias and D. T. M. Slock, "Normalized sliding window constant modulus and decision-directed algorithms: A link between blind equalization and classical adaptive filtering," IEEE Trans. Signal Processing, vol. 45, pp. 231-235, Jan. 1997.

[6] O. Tanrikulu, B. Baykal, A. G. Constantinides, and J. A. Chambers, "Constant modulus blind equalization algorithms under soft constraint satisfaction," in ICASSP'97, Munich, Germany, vol. 3, pp. 2517-2520.

[7] B. Baykal, O. Tanrıkulu, and J. A. Chambers, "Adaptive soft-constraint satisfaction (SCS) algorithms for fractionally-spaced blind equalizers," in ICASSP'97, Munich, Germany, vol. 3, pp. 853-856.

[8] O. Tanrikulu, B. Baykal, A. G. Constantinides, and J. A. Chambers, "Soft constraint satisfaction (SCS) blind channel equalization algorithms," Int. J. Adapt. Contr. Signal Process., Dec. 1997, to be published.

[9] O. Tanrikulu, A. G. Constantinides, and J. A. Chambers, "A normalized constant modulus blind equalization algorithm via constrained optimization," in Proc. Int. Conf. Telecomm., Istanbul, Turkey, Apr. 1996, vol. 2, pp. 836-839.

[10] Z. Ding, C. R. Johnson, Jr., and R. A. Kennedy, "Global convergence issues with linear blind adaptive equalisers," in Blind Deconvolution, S. Haykin, Ed. Englewood Cliffs, NJ: Prentice-Hall, 1994, pp. 60-120. des Télécommunications, May 1995. 